\title{
A modified AUGIS Delphi process to establish future research priorities in bariatric and metabolic surgery
}

Wilson, M. S. J., Knight, S., Vaughan-Shaw, P., Blakemore, A., O’Kane, M., Boyle, C., Small, P., \& Mahawar, K. (Accepted). A modified AUGIS/BOMSS Delphi process to establish research priorities in bariatric and metabolic surgery. Clinical Obesity

\section{What is already known about this subject}

There is limited data in the published literature in relation to research priority setting in the field of bariatric and metabolic surgery. We have used a modified Delphi process to engage a large number of key stakeholders in this field to determine the future research priorities specifically in bariatric and metabolic surgery.

\section{What this study adds}

- We have produced a list of research priorities in bariatric and metabolic surgery using a modified Delphi process and incorporating the views of a large number of healthcare professionals with expertise in the surgical management of weight loss.

- The list of priorities has been produced by inviting members of the bariatric and metabolic surgery multidisciplinary team to submit questions and then rank them accordingly. 


\begin{abstract}
Background

Delphi methodology may be utilised to develop consensus opinion amongst a group of experts. The aim of our study was to use a modified Delphi process to determine the future research priorities among bariatric and metabolic healthcare professionals in the United Kingdom.
\end{abstract}

\title{
Methods
}

Members of the Association of Upper Gastrointestinal Surgeons and the British Obesity and Metabolic Surgery Society (BOMSS) were invited to submit individual research questions via an online survey (phase I). Two rounds of prioritisation by multidisciplinary expert healthcare professionals (phase II and III) were completed to determine a final list of high priority research questions.

\section{Results}

Fifty-one bariatric and metabolic surgery-focused questions were identified in phase I. 35 questions were taken forward for prioritisation in phase II. Eleven high priority questions were identified in phase III. The final list of high priority questions had an emphasis on the pathophysiology and long-term sequelae of bariatric and metabolic surgery.

\section{Conclusion}

A modified Delphi process has produced a list of 11 high priority research questions in bariatric and metabolic surgery. Future studies and awards from funding bodies should reflect 
this consensus list of prioritised questions in the interest of improving patient care and encouraging collaborative research across multiple centres. 


\section{Introduction}

Bariatric surgery is now widely recognised as the most effective treatment for morbid obesity with the greatest impact on quality of life and obesity-related comorbidities (1). In addition, the most commonly performed bariatric procedures also seem to be effective for the treatment of people with type 2 diabetes with or without obesity $(2,3)$. Despite the unquestionable improvements observed following bariatric and/or metabolic surgery, there is a huge knowledge gap in the pathophysiological mechanisms underlying the clinical benefit. Thus, there is a clear need for the development of research priorities in bariatric surgery, with an emphasis on the mechanisms by which bariatric surgery works (4).

Developing a list of research priorities by consensus agreement provides an opportunity to reduce waste and add value to those who fund research in bariatric and metabolic surgery (5). Further, engaging stakeholders in setting research priorities in their own area of expertise could also encourage more meaningful multicentre research collaboration and ultimately improve the quality of research outcomes.

A modified Delphi process can be used to develop a list of priorities by consensus from a group of experts. This has been successfully utilised to develop research priorities in colorectal surgery (6), orthopaedics (7), plastic surgery (8), minimal access surgery (9) and more recently in the field of hepatobiliary surgery (10). Although a Delphi process has been used to develop a consensus statement on the one anastomosis gastric bypass (11), to our knowledge, clinical research priority setting has yet to be performed solely across the wider field of bariatric and metabolic surgery using this same process. 
We, therefore, undertook a modified Delphi process with the aim of developing a list of future research priorities for bariatric and metabolic surgery. 


\section{Methods}

A three-phased modified Delphi process was undertaken (Figure 1). This included two distinct phases of prioritisation by expert multidisciplinary stakeholders utilising established methodology, as previously described for a number of clinical projects $(6,9,10)$. Research ethic committee approval was not required for this study, as confirmed by the decision making tool on the online National Research Ethics Service website (12). Stakeholders were asked to submit questions and, thereafter, to prioritise their responses based upon their own perceived clinical need. During the prioritisation phases (II and III), only complete submissions where all questions were ranked were included in the analysis.

Phase I

Our stakeholders were the AUGIS (Association of Upper GI Surgeons of Great Britain and Ireland) membership. Members were invited by email to submit research questions across upper gastrointestinal (Upper GI) and hepato-pancreato-biliary (HPB) surgery via an online survey (http://surveymonkey.com). The social media platform Twitter was also used to broaden the awareness of the Delphi process amongst interested stakeholders. AUGIS members include both medical professionals and members of the wider multidisciplinary team. There was no limit on the number of research questions that an individual could submit. The survey was open to submissions for at least 6 weeks, with three email reminders sent to the AUGIS membership during this period.

Submitted questions were collated and then grouped into four categories: 1) HPB; 2) Benign upper GI; 3) Malignant oesophagogastric; and 4) Bariatric and metabolic surgery. Any 
disagreements regarding categorisation were resolved by consensus among the steering committee.

To assess category (4) above, a bariatric and metabolic surgery steering committee was formed where duplicate questions were removed. Questions with a similar theme were altered by consensus agreement of the steering committee. Care was taken not to alter the meaning of the reviewed questions.

Questions relating to the other three categories (HPB, benign upper GI and malignant oesophagogastric surgery) were dealt with separately by subspecialty-specific steering committees.

Phase II

Bariatric and metabolic surgery research questions were prioritised by AUGIS and BOMSS members by email invitation with a link to an online survey (Google forms). Twitter was again used to highlight the prioritisation process amongst interested stakeholders. The survey contained all of the bariatric and metabolic surgery research questions and respondents were asked to prioritise each question using a Likert scale ( 1 - lowest priority to 5 - highest priority). The survey remained open for at least 6 weeks with three email reminders sent to AUGIS members. The results were reviewed by the steering committee and a 'cut-off' point agreed by consensus, with no sight of the questions when deciding the cut-off, based on mean score $(\geq 3.0)$ following prioritisation for inclusion in the final round of prioritisation.

Phase III 
A final round of prioritisation was performed after AUGIS members were again invited by email and Twitter to follow a link to a Google forms survey and prioritise the questions using the same Likert scale as in Phase II. The survey remained open for at least 6 weeks and three email reminders were sent. Results were reviewed by the steering committee to identify the final list of prioritised questions. The criteria for inclusion in the final list of research priorities was a mean score of $\geq 3.6$ with a Likert score of $4-5$ by $\geq 60$ per cent of respondents as agreed by the steering committee. The cut-off point was agreed without sight of the questions.

Steering committee

The bariatric and metabolic surgery steering committee consisted of an Upper GI surgical registrar (MW), a bariatric dietitian (MO), two consultant bariatric surgeons (KM and PS), a lay representative $(\mathrm{CB})$ and a patient and public representative $(\mathrm{AB})$. The overall role of the steering committee was to ensure relevance of the submitted questions from both a clinical and patient perspective and to provide consensus agreement. 


\section{Results}

Four hundred and twenty seven research questions were submitted by 140 AUGIS members in phase I, representing $47.6 \%$ of the membership (Figure 2). Subspecialisation bariatric and metabolic surgery interest was declared by $52(37.1 \%)$. Once duplicated and similar questions amended or removed by consensus agreement, 51 questions were moved forward for prioritisation in phase II. Fifty-four stakeholders took part in prioritisation of the questions during phase II. An analysis of the prioritisation was performed by the steering committee and consensus reached regarding a cut-off for inclusion (mean $\geq 3.0$ ) in phase III. Thirty-five questions were included in the final phase of prioritisation and 45 stakeholders took part. Following review by the steering committee with consensus agreement on the criteria for inclusion on the final list of clinical priorities as detailed in the methods section, 11 questions were included on the final list of bariatric and metabolic surgery questions with high research priority (Figure 3). The questions which failed to make the final list of research priorities from phase III can be seen in Figure 4.

In the final round of prioritisation submissions were received from 6 (13.4\%) dietitians, $1(2.2 \%)$ nurse and $38(84.4 \%)$ surgeons. 


\section{Discussion}

Our modified Delphi process has yielded a list of 11 high priority bariatric and metabolic surgery focused research questions. This was achieved by utilising the collective expertise and views of the AUGIS membership and its subspecialty arm the British Obesity and Metabolic Surgical Society (BOMSS). This work was undertaken as part of a wider project to develop the research priorities in the field of Upper GI surgery (also incorporating the subspecialty interests of HPB, benign upper GI and malignant oesophagogastric surgery).

Our list has an emphasis on developing a greater understanding of the pathophysiology of the various bariatric and metabolic procedures as well as the long-term sequelae of bariatric and metabolic surgery. Interestingly, given the delivery of healthcare in the UK, largely through the NHS, there was also an emphasis placed upon determining best practice for targeting those who would obtain the greatest benefit from bariatric and metabolic surgery. Finally, the novel procedure one anastomosis bypass resulted in two high priority questions that related to the long-term outcomes following this surgery.

The ongoing By-Band-Sleeve study may have influenced the final list of research priorities (13). This RCT aims to compare gastric band, gastric bypass and gastric sleeve procedures and determine which is the optimal procedure in terms of weight loss, quality of life and long-term complication profile. It could, therefore, provide explanation as to why some questions failed to meet the final list of high priority research questions. When prioritising questions, some may have felt that since the trial was ongoing a number of the proposed questions would be answered and, therefore, scored questions related to this study lower than other questions they felt were not covered by this study. 
The majority $(84.4 \%)$ of those who took part in the prioritisation rounds of this study were bariatric and metabolic surgeons. This may go some way to explaining why there is a heavy slant on surgical outcomes in the list of research priorities. It is unclear whether the research priorities that we present in this manuscript definitively represents the research priorities in bariatric and metabolic surgery given the focus on purely surgical topics. This could of course represent a generalised lack of knowledge of the wider 'non-surgical' issues related to the pathophysiology and management of obesity. Members of the wider multidisciplinary team may have different priorities. Dietitians for example are likely to have prioritised a question such as 'What nutritional markers do we need to monitor post-operatively following bariatric/metabolic surgery?' or 'Which multi-vitamins and mineral supplements are required following sleeve gastrectomy surgery and at which dose?'.

This survey was publicised on Twitter and therefore members of the public were able to participate. We had no submissions from lay members of the public or patients who have been exposed to bariatric surgery. We did have lay (CB) and patient (AB) representation on the steering committee for this study. Both $\mathrm{CB}$ and $\mathrm{AB}$ were involved in the analysis, discussion and agreement of methodology during phase II and III of the process. However, further studies are required to determine patient-centred outcome measures in bariatric and metabolic surgery, and to determine what patients consider the research priorities to be. Our study has a heavy clinical bias with input primarily from bariatric surgeons and academics with specialty specific skills in bariatric surgery. We also failed to have submissions from bariatric physicians and they too may have different priorities. 
Further limitations to this study may also be the perceived low response rates from AUGIS members. Response rates in phase I across all specialties was $47.6 \%$. However, this study had a particular bariatric and metabolic surgery focus. The relevant bariatric subspecialty association affiliated to AUGIS is BOMSS with 182 consultant members. Therefore, in phase II and III response rates were $29.7 \%$ and $24.7 \%$ respectively. Despite this, other similar studies have reported findings with lower response rates ranging from 11 to $25 \%(6,8)$. Therefore, we conclude that there was sufficient engagement from the AUGIS/BOMSS membership to support the validity of our questions as a true representation of research priorities among bariatric and metabolic surgeons.

The final list of high priority research questions will be shared with funding bodies, with the aim of engaging AUGIS/BOMSS members to consider answering questions in their future clinical and academic efforts. The final list of questions should set the research agenda in bariatric and metabolic surgery and future efforts should focus on addressing these questions with collaboration between centres.

Using a modified Delphi process to determine research priorities by engaging a target population of experts is well recognised in the published literature. More recently there has been greater emphasis on involving patients to determine where future research should be focused. Incorporating patient views and ensuring adequate patient representation therefore did pose the greatest challenge in our study and aside from the patient representatives on our steering committee we had minimal input from patients with a knowledge of bariatric surgery. Future studies should seek to address this imbalance. Engaging with clinicians and other members of the multidisciplinary team was relatively straightforward, but there is no 
clear methodology to determine how to equitably incorporate the views of those who deliver bariatric and metabolic surgery healthcare versus the service users who seek to access it.

In summary, we have used a modified Delphi process to determine the research priorities in bariatric and metabolic surgery, involving a large body of stakeholder surgeons across multiple centres in the UK. There was an emphasis on prioritising research into the pathophysiology of the key bariatric and metabolic surgical procedures as well as the longterm sequelae following bariatric and metabolic surgery. Future research projects should be focused towards addressing these issues, and this would be best achieved in a multicentre format. 


\section{Conflicts of Interest Statement}

All authors have no conflicts of interest to declare.

\section{Acknowledgements and author contributions}

The bariatric and metabolic surgery steering committee wish to formally thank AUGIS and BOMSS for their support with this study. In particular, the administrative support provided by Nichola Bartlett was fundamental throughout the Delphi process.

MSJW, SK and PVS designed and the study and were involved in phase I and have had critical input in the analysis of results, review and approval of the manuscript. MSJW delivered phase II and III. AB, CB, MO, KM and PS were all invited experts who provided critical input to the study from the end of phase I. They formed part of the steering committee and with consensus enabled the completion of phase II and III. All authors have reviewed and made significant editorial contributions to this manuscript. 


\section{References}

1. Colquitt JL, Pickett K, Loveman E, Frampton GK. Surgery for weight loss in adults. Cochrane Database Syst Rev. 2014(8):CD003641.

2. Mingrone G, Panunzi S, De Gaetano A et al. Bariatric-metabolic surgery versus conventional medical treatment in obese patients with type 2 diabetes: 5 year follow-up of an open-label, single-centre, randomised controlled trial. Lancet. 2015;386(9997):964-73.

3. Muller-Stich BP, Senft JD, Warschkow R et al. Surgical versus medical treatment of type 2 diabetes mellitus in nonseverely obese patients: a systematic review and metaanalysis. Ann Surg. 2015;261(3):421-9.

4. Carlson MA. Research priorities in bariatric surgery: misplaced emphasis on innovation? Ann Surg. 2015;261(2):e58-9.

5. Chalmers I, Bracken MB, Djulbegovic $B$ et al. How to increase value and reduce waste when research priorities are set. Lancet. 2014;383(9912):156-65.

6. Tiernan J, Cook A, Geh I et al. Use of a modified Delphi approach to develop research priorities for the association of coloproctology of Great Britain and Ireland. Colorectal Dis. 2014;16(12):965-70.

7. Eubank BH, Mohtadi NG, Lafave MR et al. Using the modified Delphi method to establish clinical consensus for the diagnosis and treatment of patients with rotator cuff pathology. BMC Med Res Methodol. 2016;16:56.

8. Henderson J, Reid A, Jain A. Use of a modified BAPRAS Delphi process for research priority setting in Plastic Surgery in the UK. J Plast Reconstr Aesthet Surg. 2018;71(12):167981.

9. Francis N, Kazaryan AM, Pietrabissa A et al. A research agenda for the European Association for Endoscopic Surgeons (EAES). Surg Endosc. 2017;31(5):2042-9.

10. Knight SR, Pathak S, Christie A et al. Use of a modified Delphi approach to develop research priorities in HPB surgery across the United Kingdom. HPB (Oxford). 2019.

11. Mahawar KK, Himpens J, Shikora SA et al. The First Consensus Statement on One Anastomosis/Mini Gastric Bypass (OAGB/MGB) Using a Modified Delphi Approach. Obes Surg. 2018;28(2):303-12.

12. NHS Health Research Authority [Available from: http://www.hradecisiontools.org.uk/ethics/index.html.

13. Rogers CA, Reeves BC, Byrne J et al. Adaptation of the By-Band randomized clinical trial to $B y$-Band-Sleeve to include a new intervention and maintain relevance of the study to practice. Br J Surg. 2017;104(9):1207-14. 
Table and Figure Legends

Figure 1: The modified Delphi process used to prioritise research questions in bariatric and metabolic surgery

Figure 2. Overview of responses during modified Delphi process

Figure 3. Research priorities for bariatric and metabolic surgery

Figure 4. Questions from phase III that were not included in the final list of research priorities 